\title{
On Continued Fractions and Their Applications
}

\author{
Zakiya M. Ibran*, Efaf A. Aljatlawi, Ali M. Awin \\ Department of Mathematics, Faculty of Science, University of Tripoli, Tripoli, Libya \\ Email: awinsus@yahoo.com
}

How to cite this paper: Ibran, Z.M., Aljatlawi, E.A. and Awin, A.M. (2022) On Continued Fractions and Their Applications. Journal of Applied Mathematics and Physics, 10, 142-159.

https://doi.org/10.4236/jamp.2022.101011

Received: December 14, 2021

Accepted: January 18, 2022

Published: January 21, 2022

Copyright $\odot 2022$ by author(s) and Scientific Research Publishing Inc. This work is licensed under the Creative Commons Attribution International License (CC BY 4.0).

http://creativecommons.org/licenses/by/4.0/

\begin{abstract}
Continued fractions constitute a very important subject in mathematics. Their importance lies in the fact that they have very interesting and beautiful applications in many fields in pure and applied sciences. This review article will reveal some of these applications and will reflect the beauty behind their uses in calculating roots of real numbers, getting solutions of algebraic Equations of the second degree, and their uses in solving special ordinary differential Equations such as Legendre, Hermite, and Laguerre Equations; moreover and most important, their use in physics in solving Schrodinger Equation for a certain potential. A comparison will also be given between the results obtained via continued fractions and those obtained through the use of well-known numerical methods. Advances in the subject will be discussed at the end of this review article.
\end{abstract}

\section{Keywords}

Continued Fraction, Equation, Numerical Method, Roots, Series, Finite

\section{Introduction}

The subject of continued fractions (CF) is an old subject although many people are not aware of it. Actually, continued fractions have so many applications in algebra and in various fields such as mathematics, physics, and chemistry [1].

The easiest way of forming a continued fraction is by writing a certain amount in the form of a numerator and a denominator, and each denominator is composed of a numerator and a denominator and so on. Usually, the successive numerators are equal to one.

Continued fractions have a long history; they were known since the appearance of Euclidean algorithm for finding the greatest common divisor (GCD) of two numbers. That was around the year 300 B.C. [1] [2]. Research works and ${ }^{\star}$ Deceased about two years ago. 
papers continue then to be performed and a huge accumulation of applications arise; this is due to their simplicity to deal with and the smooth way of the calculations involved, because all what we need are the four simple mathematical operations, namely addition, subtraction, multiplication, and division.

We should add that the subject of continued fractions is still very fruitful and interesting for researchers. In fact, their uses are clear in many applied areas, in mathematics, physics, chemistry, and in medical sciences.

Somewhat recently, a short article was written about the history of continued fractions presenting research works on them and their use in power series fields over a finite field [3]. Hence, we see the motive behind writing this review article.

In the next section, we introduce continued fractions and give few examples to clarify concepts and ideas which are related to them showing how to write an ordinary fraction in the form of a continued one [4] [5] [6]. To add, we show the relationship of continued fractions with Fibonacci numbers, series and recurrence formulae [1].

In section 3 and section 4 , we give some applications in mathematics which include ways of computing roots of real numbers [7], and representation and solution of algebraic Equation of the second degree in one unknown [5] [7]. We also make a comparison between the results obtained via continued fractions techniques and the results obtained through the use of some numerical methods such as the general method to compute the $n^{\text {th }}$ root of real numbers [8], and Newton-Raphson method [9].

Using continued fractions in solving differential Equations such as Legendre, Hermite, and Laguerre Equations is the subject of section 5 [10]. Section 6 deals with applications of continued fractions in quantum mechanics in solving the time-dependent Schrodinger Equation [11]. In section 7, we discuss the convergence of CFs.

Finally, and in the last section, we present a concluding discussion.

\section{Preliminaries}

\section{Definition 1}

An expression of the form

$$
a_{1}+\frac{b_{1}}{a_{2}+\frac{b_{2}}{a_{3}+\frac{b_{3}}{\ddots}}}
$$

Is called a continued fraction; and where $a_{i}, i=1,2,3, \cdots$ and $b_{i}, i=1,2,3, \cdots$ are real numbers.

If it happens that the numerator in each fraction is equal to one, then the continued fraction is a simple one. i.e. $a_{1}+\frac{1}{a_{2}+\frac{1}{a_{3}+\frac{1}{a_{4}+\cdots}}}$ is a simple continued 
fraction [1].

Note that any normal fraction $\left(\frac{p}{q}, p\right.$ and $q$ are integers and $\left.q \neq 0\right)$ can be expressed as a continued fraction as [2]

$$
\frac{p}{q}=a+\frac{1}{b+\frac{1}{c+\cdots}} \equiv[a ; b, c, \cdots]
$$

\section{Definition 2}

The finite continued fraction in a simple one with a finite number of continued ones. i.e. it has the form

$$
\left[a_{0} ; a_{1}, a_{2}, \cdots, a_{n}\right]
$$

\section{Definition 3}

An infinite continue fraction is expressed in terms of an infinite number of continued ones; namely it has the form [2] [12]

$$
\left[a_{0} ; a_{1}, a_{2}, \cdots\right]
$$

\section{Definition 4}

An infinite continued fraction is called a periodic fraction if there exist two positive numbers $k$ and $N$ such that $a_{n}=a_{n+k} \quad \forall n \geq N$. The fraction is then written as [2]

$$
\left[a_{0} ; a_{1}, a_{2}, \cdots, a_{N}, a_{N+1}, \cdots, a_{N+k-1}, a_{N}, a_{N+1}, \cdots\right]
$$

In a compact form we write (4) as

$$
\left[a_{0} ; a_{1}, a_{2}, \cdots, \overline{a_{N}, a_{N+1}, \cdots, a_{N+k-1}}\right]
$$

Now, we see that it is easy to simplify a continued fraction to a usual fraction. This can be seen from the example below

Example 1

$$
a+\frac{b}{c+\frac{d}{f+h}}=a+\frac{b(f+h)}{c(f+h)+d}=\frac{a[c(f+h)+d]}{c(f+h)+d} .
$$

Moreover a normal fraction can always be expressed as a CF. Example 2 illustrates this process

\section{Example 2}

To express the fraction $\frac{64}{25}$ as a CF we proceed as follows

$$
\begin{gathered}
64=2 \times 25+14, \\
25=1 \times 14+11, \\
11=3 \times 3+2, \\
3=1 \times 2+1, \\
2=1 \times 2+0 .
\end{gathered}
$$

Hence it is clear that 


$$
\frac{64}{25}=2+\frac{1}{1+\frac{1}{1+\frac{1}{3+\frac{1}{1+\frac{1}{2}}}}}=[2 ; 1,1,3,1,2], \text { which is a finite CF. }
$$

What was done in this example is exactly what is to be done using Euclidean algorithm for finding the GCD of two numbers [1].

\section{Remarks}

1) If $\frac{p_{i}}{q_{i}}=\left[a_{0} ; a_{1}, a_{2}, \cdots\right]$ then $\frac{q_{i}}{p_{i}}=\left[0 ; a_{0}, a_{1}, a_{2}, \cdots\right]$ and vice versa. e.g. $\frac{45}{16}=[2 ; 1,4,3]$, then $\frac{16}{45}=[0 ; 2,1,4,3]$.

2) We call the first integer appearing in the CF as the first element in the list. 2 is the first element for the fraction $\frac{28}{11}=[2 ; 1,1,5]$; and 5 is the last element in the list for the fore-mentioned example, namely for $[2 ; 1,1,5]$.

3) To find the $\mathrm{CN}$ for a negative fraction $-\frac{p}{q}$ we search for a negative integer which when multiplied by $q$ and subtracted from $-p$ we get a smallest positive integer and the first element should be a negative number. e.g.

$$
-\frac{37}{44}=-1+\frac{1}{6+\frac{1}{3+\frac{1}{2}}}=[-1 ; 6,3,2] \text {. }
$$

4) Any rational number can be expressed in terms of a finite CF; while an irrational number can only be written as an infinite $\mathrm{CF}$ [13].

\section{Continued Fractions and Series}

Continued fractions have very good relations with various series; to begin with we start with Fibonacci series:

\subsection{Fibonacci Series}

Leonardo Fibonacci (1170-1250 A.D.) was a merchant and dealt a lot with Arab mathematicians [1]; one of the things he was famous for was his series about a couple of rabbits, male and female, giving birth to off-springs and getting after $n$ months the following series

$$
1,1,2,3,5,8,13,21,34,55,89,144,233,377,610,987, \cdots
$$

The question which needed an answer was: After $n$ months how many couples of rabbits were there? The answer was given by Fibonacci where he took into account of the fertility of the rabbits and that the numbers of couples in the $i^{\text {th }}$ month $\left(u_{i}\right)$ is the sum of the ones born in that month and the previous number counted in the $(i-1)^{\text {th }}$ month $\left(u_{i-1}\right)$ reaching the conclusion given in (6). These are Fibonacci numbers and are denoted by $u_{0}(=0), u_{1}(=1), u_{2}(=1), u_{3}(=2), u_{4}(=3), \cdots \quad[1]$. The recurrence relation 


$$
u_{i+1}=u_{i}+u_{i-1}
$$

Used before is very common in nature especially in the classification of leaves in botany and in sunflower studies [1].

Now, it clear that Fibonacci numbers can be written in terms of CF. This is shown as follows: $u_{2}=u_{1}+u_{0} \Rightarrow \frac{u_{2}}{u_{1}}=1+\frac{u_{0}}{u_{1}}$; and

$$
u_{4}=u_{3}+u_{2} \Rightarrow \frac{u_{4}}{u_{3}}=1+\frac{1}{\frac{u_{3}}{u_{2}}}=1+\frac{1}{1+\frac{1}{1+\frac{1}{\frac{u_{1}}{u_{0}}}}}
$$

\subsection{Continued Fractions in Recursive Forms}

Assume that we have the fraction

$$
\begin{aligned}
& \frac{p_{n}}{q_{n}}=\left[a_{0} ; a_{1}, a_{2}, \cdots, a_{n}\right]=a_{0}+\frac{1}{a_{1}+\frac{1}{a_{2}+\frac{1}{a_{3}+\cdots}}} \\
& \cdots+\frac{1}{a_{n}}
\end{aligned}
$$

The numerators in each of the fractions are [5]

$$
p_{0}=a_{0}, p_{1}=a_{0} a_{1}+1=a_{1} p_{0}+1, \cdots, \text { etc. }
$$

In general it can be shown that [5]

$$
p_{n}=a_{n} p_{n-1}+p_{n-2}
$$

Equation (9), gives the recurrence relations between the successive numerators of the meant fractions; while the similar recurrence relations between the successive denominators $q_{i}$ are given by

$$
q_{n}=a_{n} q_{n-1}+q_{n-2}
$$

Also it is clear that

$$
\frac{p_{n}}{q_{n}}=\frac{a_{n} p_{n-1}+p_{n-2}}{a_{n} q_{n-1}+q_{n-2}}, n \geq 2
$$

\section{Example 3}

since

$$
\begin{aligned}
& \frac{17}{12}=[1 ; 2,2,2], \therefore \frac{p_{0}}{q_{0}}=1, \frac{p_{1}}{q_{1}}=1+\frac{1}{2}=\frac{3}{2}, \frac{p_{2}}{q_{2}}=1+\frac{1}{2+\frac{1}{2}}=1+\frac{2}{5}=\frac{7}{5}, \\
& \text { and } \frac{p_{3}}{q_{3}}=1+\frac{1}{2+\frac{1}{2+\frac{1}{2}}}=1+\frac{1}{2+\frac{2}{5}}=1+\frac{5}{12}=\frac{17}{12}
\end{aligned}
$$

As expected. 


\subsection{Ascending Continued Fractions}

Although this kind of fraction is not very much used but there is no harm in defining it here at least from historical point of view [1] [4]. We can write the fraction $\frac{a}{b}$ as an Ascending continued fraction as

$$
\frac{a}{b} \equiv \frac{1}{c}+\frac{1}{d}=\frac{1}{c}+\frac{1}{c} \cdot \frac{1}{e}=\frac{1+\frac{1}{e}}{c}
$$

\section{Example 4}

To write the fraction $\frac{5}{12}$ in terms of an ascending CF, we see that

$$
\frac{5}{12}=\frac{1}{3}+\frac{1}{12}=\frac{1}{3}+\frac{1}{3} \cdot \frac{1}{4}=\frac{1+\frac{1}{4}}{3} .
$$

\section{The Use of Continued Fractions in Finding Roots of Real Numbers}

\subsection{The Square Root}

There are three methods used to compute square roots of real numbers using continued fractions [7]; we start with the first method,

\section{Method 1}

To get the square root of a certain number $A$ and if $a$ is the largest number such that its square is less than $A$; then we can compute the square root of $A$ as shown in the example below.

\section{Example 5}

To represent the irrational number $\sqrt{2}$ as a CF we see that $A=\sqrt{2}$ and $a=1$, therefore $\sqrt{2}=1+\sqrt{2}-1$. Hence we get

$$
\sqrt{2}=1+\frac{1}{\frac{1}{\sqrt{2}}-1}=1+\frac{1}{\frac{1}{\sqrt{2-1}} \times \frac{\sqrt{2}+1}{\sqrt{2}+1}}=1+\frac{1}{\sqrt{2}+1} ; \text { but } \sqrt{2}+1=2+\frac{1}{\sqrt{2}+1} \text {. }
$$

Substituting with $\frac{1}{\sqrt{2}+1}$ and repeating the substitution process, we get

$$
\sqrt{2}=1+\frac{1}{2+\frac{1}{2+\frac{1}{2+\cdots}}}=[1 ; 2,2, \cdots]=[1 ; \overline{2}] . \text { Therefore, we were able to write }
$$

$\sqrt{2}$ in terms of CFs.

\section{Method 2}

If $r=\sqrt{z}=\sqrt{a^{2}+b} \equiv a+c$, where $a$ is the greatest positive integer such that its square is less than $z$ and $b$ is the remaining i.e. $z=a^{2}+b$, then it is easy to see that $c=\frac{b}{2 a+c}$. This will be the recurrence relation we will use in the calculation to get square roots for real numbers. To begin with, we see that the first 
approximation for $\sqrt{z}$ is $r_{1}=a+c_{0}$ and the second approximation is $r_{2}=a+c_{1}=a+\frac{b}{2 a+c_{0}}$, and this gives the second approximation for the required root. The third approximation is given by

$$
\begin{aligned}
& r_{3}=a+c_{2}=a+\frac{b}{2 a+\frac{b}{2 a}} \text {. continuing, we get } \\
& \qquad r=\sqrt{z}=\sqrt{a^{2}+b}=a+\frac{b}{2 a+\frac{b}{2 a+\frac{b}{2 a+\frac{b}{2 a+\cdots}}}}
\end{aligned}
$$

This is the required recurrence relation, in terms of a $\mathrm{CN}$, for computing square roots of real numbers.

\section{Example 6}

To use Equation (14) to get $\sqrt{10}$. We see that $10=3^{2}+1$, hence $a=3$ and

$$
b=1 \text {; and } r=\sqrt{10}=\sqrt{3^{2}+1}=3+\frac{1}{6+\frac{1}{6+\frac{1}{6+\frac{1}{6+\cdots}}}} \text {; by evaluating the resulting }
$$

CF we get the approximate answer for $\sqrt{10}$.

\section{Method 3}

This method depends on partial guessing of $a$ instead of getting the greatest integer mentioned in method 2 [6].

\section{Example 7}

To clarify what we meant by partial guessing of $a$, we compute $\sqrt{2}$, using Equation (14), as follows

$$
2^{\frac{1}{2}}=\sqrt{\left(\frac{3}{2}\right)^{\frac{1}{2}}-\frac{1}{4}}=\frac{3}{2}+\frac{-\frac{1}{4}}{3+\frac{-\frac{1}{4}}{3+\frac{-\frac{1}{4}}{3+\cdots}}} \text { Table } 1 \text { shows some square roots for }
$$

certain prime integers [6].

\section{2. $n^{\text {th }}$ Roots}

Here, we will deal with the $n^{\text {th }}$ root of real numbers such that $n \geq 2$. We write $r=z^{\frac{1}{n}}=\left(a^{n}+b\right)^{\frac{1}{n}}=a+c$; then $a^{n}+b=(a+c)^{n}$. Using the binomial theorem, we get $[6]$

$$
a^{n}+b=a^{n}+n a^{n-1} c+\frac{n(n-1) a^{n-2} c^{2}}{2 !}+\frac{n(n-1)(n-2) a^{n-3} c^{3}}{3 !}+\cdots
$$

With straightforward simplifications we get 
Table 1. Square roots for certain prime numbers using CFs.

\begin{tabular}{cc}
\hline Number & Root as a CF \\
\hline 2 & {$[1 ; \overline{2}]$} \\
3 & {$[1 ; \overline{1,2}]$} \\
5 & {$[2 ; \overline{4}]$} \\
7 & {$[2 ; \overline{1,1,1,4}]$} \\
11 & {$[3 ; \overline{3,6}]$} \\
13 & {$[4 \overline{1,1,1,1,6}]$} \\
19 & {$[4 \overline{2,1,3,1,2,8}]$} \\
23 & {$[4 ; \overline{1,3,1,8}]$} \\
\hline
\end{tabular}

$$
c=\frac{b}{n a^{n-1}+\frac{n(n-1) a^{n-2} c}{2 !}+\frac{n(n-1)(n-2) a^{n-3} c^{2}}{3 !}+\cdots}
$$

Hence our first guess $c_{1}$ is

$$
c_{1}=\frac{b}{n a^{n-1}}
$$

And the first approximation for the root is

$$
r_{1}=a+c_{1}=a+\frac{b}{n a^{n-1}}
$$

Using Equation (16) and Equation (17), we get the second guess in $c$ as

$$
c_{2}=\frac{b}{n a^{n-1}+\frac{b(n-1)}{2 a}}
$$

The second approximation for $r$ is then

$$
r_{2}=a+c_{2}=a+\frac{b}{n a^{n-1}+\frac{b(n-1)}{2 a}}
$$

In general we get

$$
r=z^{\frac{1}{n}}=\left(a^{n}+b\right)^{\frac{1}{n}}=a+\frac{b}{n a^{n-1}+\frac{b(n-1)}{2 a+\frac{b(n+1)}{3 n a^{n-1}+\frac{b(2 n-1)}{2 a+\frac{b(2 n+1)}{5 n a^{n-1}+\cdots}}}}}
$$

\section{Example 8}

To express $\sqrt[5]{33}$ in terms of CN; we follow the following steps:

From Equation (21) and since $z=33, a=2, b=1$, we get 


$$
\begin{aligned}
& r=\sqrt[5]{33}=2+\frac{1}{5 \times 2^{4}+\frac{4}{2 \times 2+\frac{6}{15 \times 2^{4}+\frac{11}{2 \times 2+\frac{14}{5 \times 5 \times 2^{4}+\frac{14}{2 \times 2+\cdots}}}}}} \\
& =2+\frac{1}{80+\frac{4}{4+\frac{6}{240+\frac{9}{4+\frac{11}{400+\frac{14}{4+\cdots}}}}}}
\end{aligned}
$$

\subsection{Evaluation of Quantities in the Form $z^{\frac{m}{n}}$}

Again we put $r=z^{\frac{m}{n}}=\left(a^{n}+b\right)^{\frac{m}{n}}=a^{m}+c$, and in the same manner as in the last subsection we expand $\left(a^{m}+c\right)^{n}$ using the binomial theorem to get

$$
\left(a^{m}+c\right)^{n}=a^{m n}+n a^{m(n-1)} c+\frac{n(n-1) a^{m(n-2)} c^{2}}{2 !}+\cdots
$$

Noting that

$$
\left(a^{n}+b\right)^{\frac{m}{n}}=a^{m}+c \Rightarrow\left(a^{n}+b\right)^{m}=\left(a^{m}+c\right)^{n}
$$

And that

$$
\left(a^{n}+b\right)^{m}=a^{m n}+m a^{n(m-1)} b+\frac{m(m-1) a^{n(m-2)} b^{2}}{2 !}+\cdots
$$

From the last three Equation $s$ we see that $c$ is given by

$$
c=\frac{m a^{n(m-1)} b+\frac{m(m-1) a^{n(m-2)} b^{2}}{2 !}+m(m-1)(m-2) a^{n(m-3)} b^{3}+\cdots}{n a^{m(n-1)}+\frac{n(n-1) a^{m(n-2)} c}{2 !}+\frac{n(n-1)(n-2) a^{m(n-3)} c}{3 !}+\cdots}
$$

Directly, we obtain the first guess in $c$ and the first approximation in $r$ as

$$
c_{1}=\frac{m a^{n(m-1)} b}{n a^{m(n-1)}}=\frac{m b}{n a^{(n-m)}}, \quad r_{1}=a^{m}+\frac{m b}{n a^{(n-m)}}
$$

Following a similar procedure as in the last subsection, we obtain the general formula given by [6]

$$
\begin{aligned}
& r=z^{\frac{m}{n}}=a^{m}+c=a^{m}+\frac{m b}{n a^{n-m}+\frac{(n-m) b}{(n+m) b}}
\end{aligned}
$$

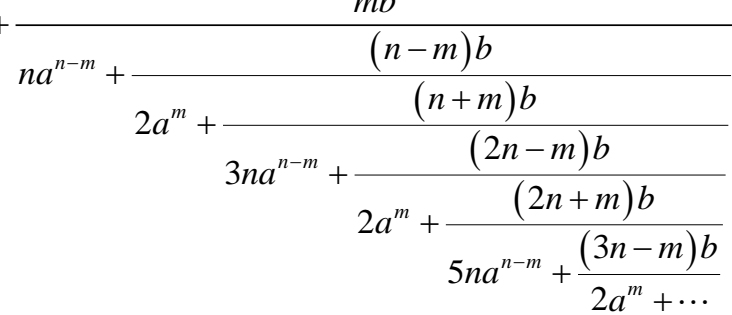




\section{Example 9}

To evaluate $r=65^{\frac{2}{3}}$ in terms of CFs, we see that $z=65, m=2, n=3$. Moreover, we have $r=65^{\frac{2}{3}}=\left(4^{3}+1\right)^{\frac{2}{3}}=4^{2}+c$.

So $\left(4^{3}+1\right)^{\frac{3}{2}}=4^{2}+c \rightarrow\left(4^{3}+1\right)^{2}=\left(4^{2}+c\right)^{3}$. This yields $c=\frac{129}{768+48 c+c^{2}}$; this is the required recurrence relation needed to accomplish the job. As a first start we take $c_{0}=\frac{129}{768}$ and $r_{1}=a^{2}+c_{0}=16+\frac{129}{768}=16.167968$. Note that the second guess for $c$ is

$$
c_{1}=\frac{129}{768+48\left(\frac{129}{768}\right)+\left(\frac{129}{768}\right)^{2}}=0.16621768 \text { and } r_{2}=a^{2}+c_{1} 16.16621768 ;
$$

and finally we get $r=65^{\frac{3}{2}}=16+\frac{2}{12+\frac{1}{32+\frac{5}{36+\cdots}}}$.

Note that another way of computing roots of real numbers is given by the Equation below and this Equation gives the root in a more precise and quicker manner as will be shown in the following example.

\section{Example 10}

To compute $\sqrt{10}$ using the Equation below

$$
r=z^{\frac{m}{n}}=\left(a^{n}+b\right)^{\frac{m}{n}}=a^{m}+c \quad \frac{2 a^{m} b m}{2 n z-b(m+n)-\frac{\left(n^{2}-m^{2}\right) b^{2}}{\left(4 n(2 z-b)-\frac{\left(4 n^{2}-m^{2}\right) b^{2}}{5 n(2 z-b)-\frac{\left(16 n^{2}-m^{2}\right) b^{2}}{9 n(2 z-b)+\cdots}}\right.}}
$$

where $r, z, a, b, c, n, m$ are as before.

Here, we see that $z=10, a=3, b=1, n=2, m=1$; and substituting in Equation (28) we obtain

$$
r=3+\frac{6}{37-\frac{3}{114-\frac{15}{190-\frac{35}{266-\cdots}}}} \text {. Now we see that as a first approximation we }
$$

get 3 , the second one is $3.162162162162 \ldots$ and this correct up to three decimal figures; the third approximation is $3.16227758007117 \ldots$, it is correct up to 6 decimal points; the fourth one is $3.16227766011283 \ldots$, and so on.

\subsection{Comparison between the Use of Continued Fractions and Some Other Numerical Methods in Computing Roots}

In this subsection, we make a comparison between the method of CFs (CFM) 
and two other numerical methods, namely the general method of computing $n^{\text {th }}$ roots of real numbers (GM) [8] and the well-known Newton-Raphson method (NRM) for the same purpose. Just for the sake of comparison [6] [8] [9], we give few examples in Table 2.

Note that the GM is very exact in the sense that whatever digit we get from the root is solid and no further modification in it once we get it; while in the other two methods various iterations improve the accuracy in the root. In the use of CFM we needed ten steps of Approximations to reach the accurate value of $\sqrt{2}$ shown in Table 2; and five approximations were needed to get the value of $\sqrt[3]{56423456}$.

As to the use of NRM we have to make five iterations to get the same answers for both roots. Moreover, a software has to be used for the calculation of the roots for the two methods CNM and NRM. However, the GM, although a software can be developed for the calculation, but with patience the value can be obtained by using a pen and a pencil and every digit one gets from the root is exact and final [8].

The GM was obtained by experience and the theory behind it is still unknown. The theory may lead to an inverse binomial theorem.

\section{Solutions of Algebraic Equations Using Continued Fractions}

The algebraic Equation of the second degree in one variable is given by

$$
a x^{2}+b x+c=0, a \neq 0
$$

It is a common practice to get the roots of the last Equation s via completion of the square method or using the general rule which gives the two roots as

$$
x_{ \pm}=\frac{-b \pm \sqrt{b^{2}-4 a c}}{2 a}
$$

However, these methods yield, in general, irrational answers; therefore we refer to CF method to get approximate roots in a rational form.

\section{Example 11}

To solve the Equation $x^{2}-2=0$ we see that $x^{2}-1=1$; or $(x-1)(x+1)=1$, from which we obtain $x=1+\frac{1}{x+1}$. Hence we get

$$
x=1+\frac{1}{1+\left(1+\frac{1}{1+x}\right)}=1+\frac{1}{2+\frac{1}{1+x}} \text {; we can continue this process to get the }
$$

required root via CFs; we note that even with the few terms we have written and if we take the first guess as 1 then the first approximation is given by $x_{1}=1+\frac{1}{2+1 / 2}=\frac{7}{5}=1.4$ which is a rational answer and is correct up to one decimal point. It is a crude answer, but acceptable for such one iteration.

Now, going back to Equation (30) we see that 
Table 2. A comparison between CFM, GM, and NRM for Computing the values of $\sqrt{2}$ and $\sqrt[3]{56423456}$.

\begin{tabular}{cccc}
\hline Number & CNM & GM & NRM \\
\hline$\sqrt{2}$ & 1.4142136248 & 1.41421356237 & 1.41421356 \\
$\sqrt[3]{56423456}$ & 383.54815 & 383.54815 & 383.54815 \\
\hline
\end{tabular}

$$
x=-b-\frac{c}{x}, a \text { is put as } 1
$$

And hence the root is given by

$$
x=-b-\frac{c}{-b-\frac{c}{-b-\frac{c}{-b-\frac{c}{-b-\cdots}}}}
$$

\section{Example 12}

Consider the quadratic Equation $x^{2}-5 x-6=0$, then using

Equation (32) we see that the root is given by $x=5+\frac{6}{5+\frac{6}{5+\frac{6}{5+\cdots}}}$, and if we

take the initial guess as $x_{0}=5$, we can compute one of the roots of the given Equation using four approximations as $x \cong 5.999 \cong 6$.

\section{Continued Fractions and Their Use in Special Ordinary Differential Equations}

In this section, we show how to use CNs in solving special ordinary differential Equation s leading to special functions; we start with Hermite differential Equation [10].

\subsection{Hermite Differential Equation}

Hermite differential Equation has the form

$$
\ddot{y}-2 x \dot{y}+2 \alpha y=0
$$

From which we obtain

$$
\frac{\mathrm{d} \ln y}{\mathrm{~d} x}=\frac{\dot{y}}{y}=\frac{2 \alpha}{2 x-\frac{\ddot{y}}{\dot{y}}}
$$

Differentiating Equation (33) and with few simplifications, we get

$$
\frac{\mathrm{d} \ln y}{\mathrm{~d} x}=\frac{\dot{y}}{y}=\frac{2 \alpha}{2 x-\frac{2(\alpha-1)}{2 x-\frac{\dddot{y}}{\ddot{y}}}}
$$

Again differentiating Equation (33) twice we get 


$$
\frac{\mathrm{d} \ln y}{\mathrm{~d} x}=\frac{\dot{y}}{y}=\frac{2 \alpha}{2 x-\frac{2(\alpha-1)}{2 x-\frac{2(\alpha-2)}{2 x-\frac{y^{\mathrm{iv}}}{\dddot{y}}}}}
$$

Now, $\alpha$ can be $\alpha=0,1,2,3, \cdots ;$ e.g. if $\alpha=0$ then $\frac{\mathrm{d} \ln y}{\mathrm{~d} x}=0 \Rightarrow y=\mathrm{constant}=c$, while if $\alpha=1$ then $y=c x$ and if $\alpha=2$ then $y=c\left(2 x^{2}-1\right)$,...etc. [8]. These polynomials are the first few Hermite polynomials with $c$ taking an appropriate value [10].

\subsection{Legendre Differential Equation}

Legendre differential Equation is given by

$$
\left(1-x^{2}\right) \ddot{y}-2 x \dot{y}+l(l+1) y=0
$$

Following the same steps as in the previous subsection we obtain

$$
\frac{\mathrm{d} \ln y}{\mathrm{~d} x}=\frac{\dot{y}}{y}=\frac{l(l+1)}{2 x-\left(1-x^{2}\right) \frac{\ddot{y}}{\dot{y}}}
$$

Differentiating Equation (36) and with some simplifications we get

$$
\frac{\mathrm{d} \ln y}{\mathrm{~d} x}=\frac{\dot{y}}{y}=\frac{l(l+1)}{2 x-\left(1-x^{2}\right) \frac{l(l+1)-2}{4 x-\left(1-x^{2}\right) \frac{\dddot{y}}{\ddot{y}}}}
$$

Moreover, it can be shown that

$$
\frac{\mathrm{d} \ln y}{\mathrm{~d} x}=\frac{\dot{y}}{y}=\frac{l(l+1)}{2 x-\left(1-x^{2}\right) \frac{l(l+1)-2}{4 x-\left(1-x^{2}\right) \frac{l(l+1)-6}{6 x-\left(1-x^{2}\right) \frac{y^{\mathrm{iv}}}{\dddot{y}}}}}
$$

In the same manner shown in the last subsection we put $l=0,1,2$ to obtain the first few Legendre polynomials which are $y=c, c x, c\left(3 x^{2}-1\right)$ respectively, of course with an appropriate choice of the constant $c[10]$.

\subsection{Laguerre Differential Equation}

The Equation is given by

$$
x \ddot{y}+(1-x) \dot{y}+\alpha y=0
$$

Repeating the same procedure followed in the last subsection we get

$$
\frac{\mathrm{d} \ln y}{\mathrm{~d} x}=\frac{\dot{y}}{y}=\frac{\alpha}{(x-1)-x \frac{\alpha-1}{(x-2)-x \frac{\alpha-2}{(x-3)-x \frac{y^{\mathrm{iv}}}{\dddot{y}}}}}
$$


Putting $\alpha=0,1,3$ to obtain $y=c, c(x-1), c\left(x^{2}-4 x+2\right)$ respectively and which are the first three Laguerre Polynomials with the right choice of the constant $c[10]$.

\subsection{Chebyshev Differential Equation}

Chebyshev differential Equation is expressed as

$$
\left(1-x^{2}\right) \ddot{y}-x \dot{y}+p^{2} y=0
$$

In the same way followed in the previous subsections we get

$$
\frac{\mathrm{d} \ln y}{\mathrm{~d} x}=\frac{\dot{y}}{y}=\frac{p^{2}}{x-\left(1-x^{2}\right) \frac{\ddot{y}}{\dot{y}}}
$$

Further, we obtain

$$
\frac{\mathrm{d} \ln y}{\mathrm{~d} x}=\frac{\dot{y}}{y}=\frac{p^{2}}{x-\left(1-x^{2}\right) \frac{p^{2}-1}{3 x-\left(1-x^{2}\right) \frac{p^{2}-4}{2 x-\left(1-x^{2}\right) \frac{y^{\text {iv }}}{\dddot{y}}}}}
$$

Now, putting $p=0,1,2$ we get the first three Chebyshev polynomials $y=c, c x, c\left(2 x^{2}-1\right)$ respectively; with consideration given to the appropriate value of $c$.

Note that a comparison between the use of CFs and Special Bilinear functions (SBF), in computing special functions, is in order. Both are interesting but the use of SBF is more direct and illustrative; in fact SBF procedure gives the right polynomials directly and moreover it leads to recurrence relations between these polynomials [14] [15].

\section{Continued Fractions and the Solution of the Time-Dependent Schrodinger Equation}

In this application of CFs, we show how CFs are used to get the solution of the time-dependent Schrodinger Equation [11]; the obtained formula will enable us to take care of the problem of time variation.

Assume that a particle (an electron say) moves on Bethe lattice with a Hamiltonian $\mathbb{H}$ given by

$$
\mathbb{H}=\mathcal{E}|0\rangle\langle 0|+\sum_{\langle i, j\rangle} T(|i\rangle\langle j|+| j\rangle\langle i|)
$$

where $\langle i \mid j\rangle$ is the nearest neighbor to Bethe lattice, $T$ is the transpose matrix between the two states $i$ and $j$, and $\mathcal{E}$ is the lowest energy of the particle.

Now we proceed with the solution of Schrodinger Equation. The time-dependent Schrodinger Equation is described as

$$
i \frac{\partial|\Psi(t)\rangle}{\partial t}=\mathbb{H}|\Psi(t)\rangle
$$


$\mathbb{H}$ is the Hamiltonian, $\hbar \equiv 1$, and $\Psi$ is the wave function. We use the method of eigenfunction expansion to write $\Psi$ as

$$
\| \Psi(t)\rangle=\sum_{n \geq 0} c_{n}(t)\left|f_{n}\right\rangle
$$

$\left|f_{n}\right\rangle, n=0,1,2,3, \cdots$ form a basis of orthogonal functions and $c_{n}(t)$ are dependent functions on time.

Now, if $\left|f_{0}\right\rangle$ is chosen then from Gram-Schmidt algorithm, we can build the rest of the members of the basis as follows

$$
\left|f_{1}\right\rangle=H\left|f_{0}\right\rangle-a_{0}\left|f_{0}\right\rangle
$$

$a_{0}$ is taken such that $\left\langle f_{0} \mid f_{1}\right\rangle=0$; i.e.

$$
a_{0}=\left\langle f_{0}|\mathbb{H}| f_{0}\right\rangle /\left\langle f_{0} \mid f_{0}\right\rangle
$$

In the same way we put

$$
\left|f_{2}\right\rangle=H\left|f_{1}\right\rangle-a_{1}\left|f_{1}\right\rangle-b_{1}\left|f_{0}\right\rangle
$$

The orthogonality of the three functions will yield

$$
a_{1}=\left\langle f_{1}|\mathbb{H}| f_{1}\right\rangle /\left\langle f_{1} \mid f_{1}\right\rangle
$$

while $b_{1}$ is given by

$$
b_{1}=\left\langle f_{1} \mid f_{1}\right\rangle /\left\langle f_{0} \mid f_{0}\right\rangle
$$

In general, we have

$$
\left|f_{n+1}\right\rangle=H\left|f_{n}\right\rangle-a_{n}\left|f_{n}\right\rangle-b_{n}\left|f_{n-1}\right\rangle
$$

where

$$
a_{n}=\left\langle f_{n}|\mathbb{H}| f_{n}\right\rangle /\left\langle f_{n} \mid f_{n}\right\rangle
$$

And

$$
b_{n}=\left\langle f_{n} \mid f_{n}\right\rangle /\left\langle f_{n-1} \mid f_{n-1}\right\rangle
$$

Note that $\left|f_{-1}\right\rangle \cong 0$ and $b_{0} \cong 0$, by definition.

From Equation (47) and Equation (48) we see that

$$
i \sum_{n \geq 0} \frac{\mathrm{d} c_{n}(t)}{\mathrm{d} t}\left|f_{n}\right\rangle=H \sum_{n \geq 0} c_{n}(t)\left|f_{n}\right\rangle
$$

From Equation (54) and the last Equation we get

$$
i \frac{\mathrm{d} c_{n}(t)}{\mathrm{d} t}=b_{n+1} c_{n+1}(t)+a_{n} c_{n}(t)+c_{n-1}(t)
$$

where $c_{-1}(t)=0$ and the initial conditions imply that $c_{0}(t=0)=1$ and $c_{n}(t=0)=0 \quad \forall n>0$.

Now the Laplace Transform of $c_{n}(t)$ is given by

$$
\tilde{c}_{n}(z)=\int_{0}^{\infty} \mathrm{e}^{-z t} c_{n}(t) \mathrm{d} t=z \tilde{c}_{n}(z)-c_{n}(0)
$$

With further elaboration on the evaluations of various approximations, we get in its final form as 


$$
\tilde{c}_{n}(z)=\frac{1}{z+i a_{0}+\frac{b_{1}}{z+i a_{1}+\frac{b_{2}}{z+i a_{2}+\cdots}}}
$$

Once we get $\tilde{c}_{n}(z)$, we can evaluate $c_{0}(t)$ using inverse Laplace transform [9]. Note that $a_{n}$ and $b_{n}, n=0,1,2,3, \cdots$ in Equation (60) are very essential in taking the change in time into account.

\section{Convergents and Convergence of Continued Fractions}

The following notations can also be used for CNs [16]

$$
\left[a_{0},\left(b_{0}, a_{1}\right),\left(b_{1}, a_{2}\right), \cdots\right]=a_{0}+\frac{b_{0}}{a_{1}+\frac{b_{1}}{a_{2}+\frac{b_{2}}{\ldots}}}
$$

And if we write

$$
\begin{array}{r}
{\left[a_{0},\left(b_{0}, a_{1}\right),\left(b_{1}, a_{2}\right), \cdots,\left(b_{n-1}, a_{n}\right)\right]=a_{0}+\frac{b_{0}}{a_{1}+\frac{b_{1}}{a_{2}+\frac{b_{2}}{\ddots}}}} \\
a_{n-1}+\frac{b_{n-1}}{a_{n}}
\end{array}
$$

Then the truncated CF in Equation (71) is called the $n^{\text {th }}$ convergent of the CF in Equation (70) [16].

Note that the value of a finite CF can be obtained as a rational number; while the value of an infinite CF can be calculated in an approximate manner and if the limit in the Equation given below exists.

$$
\left[a_{0},\left(b_{0}, a_{1}\right),\left(b_{1}, a_{2}\right), \cdots\right]=\lim _{n \rightarrow \infty}\left[a_{0},\left(b_{0}, a_{1}\right),\left(b_{1}, a_{2}\right), \cdots,\left(b_{n-1}, a_{n}\right)\right]
$$

Then, we say that the infinite CF convergent [16].

Several recursive formulae and properties were discussed and theorems proved about CFs in the above thesis. Moreover, Harmonic CFs were introduced and the use of CFs in developing efficient algorithms that can break public-key cryptosystems, which are the backbone of internet secure communication, was illustrated [16]. For more information about convergents and CFs convergence one can refer to Reference [12].

\section{Concluding Discussion}

The subject of continued fractions is still vital in many fields. It can help in establishing an efficient algorithm to evaluate Y's functions in space dynamics; the algorithm is valid to be used for any conic section [17].

Also, CFs can be used to organize, as a new theoretical aspect, Euclidean algorithm for finding the GCD of two numbers with the help of a pseudocode [18]; the code is independent of programming languages and is universal in the sense 
that it can be transformed into solutions which lead to important applications of CFs with a new approach. The benefits behind that are the usefulness for specialists and teachers in the fields of informatics, mathematics, and parallel computations [18].

Another application of CFs is studying double-sided CFs, with coefficients which are non-commutative symbols, and their relation with the theory of discrete integrable systems [19].

In quantum mechanics, there is another application for CFs in Probing Schrodinger Equation where a continued fraction potential was used to search for possible solutions of the Equation [20].

A very recent work on CFs is an MA thesis published electronically in December 2021, which showed the continuous interest in the subject of continued fractions and their applications in a variety of fields of mathematics such as number theory and abstract algebra [2]. One of the interesting applications of CFS is their use in obtaining expressions for functions such as $\tan x$ and the evaluation of certain numbers, e.g. $\frac{4}{\pi} \quad[2]$.

Even in the complex field, continued fractions play an important role in conjunction with the evaluation of binary quadratic forms [21].

One can continue with presenting the so many applications of CFs and that will take a huge amount of work to accomplish the job, but we shall give here one more application and consider it as a final one. The application has to do with folding; if we repeat folding a strip of paper in half and unfolding it in straight angles, then we get a fractal which is known as the dragon curve. The sequence of right and left turns is related to a CF which constitutes a simple infinite series; so many properties and functions may arise from that leading to a shape resembling the dragon curve [22].

\section{Conflicts of Interest}

The authors declare no conflicts of interest regarding the publication of this paper.

\section{References}

[1] Brezinski, C. (2010) The Italian Contribution to the Foundation and Development of Continued Fractions. Rendiconti del Seminario Matematico Universita e Politecnico di Torino, 68, 1-16.

[2] Parrish, K.L. (2021) A Study in Applications of Continued Fractions. M.A. Thesis, California State University, San Bernardino. https://scholarworks.lib.csusb.edu/etd/1371

[3] Lasjaunias, A. (2017) A Short History of Some Recent Research on Continued Fractions in Function Fields. Technical Report, University of Bordeaux, Bordeaux, 1-7.

[4] Widz, J. (2009) From the History of Continued Fractions. WDS09, Proceeding of Contributed Papers, Part I, Prague, 2-5 June 2009, 176-181.

[5] Siverman, V.H. (2012) A Friendly Introduction to Number Theory. 4th Edition, Pearson Education Ltd., Essex. http://www.math.brown.edu./ jhs/frint.html 
[6] Olds, C.D. (1963) Continued Fractions. Random House and L.W. Singer Company, New York. https://www.ms.uky.edu>files

[7] Sardina, M. (2007) General Method for Extracting Roots Using Folded Continued Fractions. Surrey.

[8] Awin, A.M. (1982) A General Method to Compute the nth Root of Real Numbers. International Journal of Mathematical Education in Science and Technology, 13, 139-142. https://doi.org/10.1080/0020739820130203

[9] Ibran, Z.M. (2014) Continued Fractions and Their Applications. M.Sc. Thesis, University of Tripoli, Tripoli.

[10] David, C.W. (2009) Continued Fraction Solutions to Hermite, Legendre, and Laguerre Differential Equations. Chemistry Education Materials, 77, 1-7.

https://opencommons.uconn.edu/chem edu/77

[11] Kim, J. and Chang, I. (2001) Exact Solution of the Time-Dependent Schrodinger Equation for the Bethe Lattice in the Presence of an Impurity. Journal of the Korean Physical Society, 39, L952-L955.

[12] Baumann, H. (2019) Generalized Continued Fractions: A Definition and a Pringsheim-Type Convergence Criterion. A Springer Open Journal, 406, 1-30.

https://doi.org/10.1186/s13662-019-2340-9

[13] Guerzhoy, P. (1964) A Short Introduction to Continued Fractions. In: Khinchin, A.Y., Eds., Continued Fractions, Seventh Edition, The University of Chicago Press, Chicago. http://www.math.temple.edu/ pasha/contfrac.pdf

[14] Awin, A.M. (2015) The Use of Special Bilinear Functions in Computing Some Special Functions. Journal of Progressive Research in Mathematics, 5, 437-443.

[15] Juroud, A.A., Sharif, B.W. and Awin, A.M. (2021) Special Trilinear Functions with Few Applications. Journal of Applied Mathematics and Physics, 9, 2698-2705.

https://www.scirp.org/journal/jamp

https://doi.org/10.4236/jamp.2021.911173

[16] Tonien, J. (2018) Continued Fractions and Their Applications. Ph.D. Thesis, University of Wollongong, Wollongong.

[17] Sharaf, A.M., Saad, A.S. and Abd El Motelp, N.S. (2015) Continued Fraction Evaluation of the Universal Y's Functions. International Journal of Astronomy and Astrophysics, 5, 15-19. http://www.scirp.org/journal/ijaa

https://doi.org/10.4236/ijaa.2015.51003

[18] Iliev, A. and Kyurkchiev, N. (2019) New Organizing of the Euclid's Algorithm and One of Its Applications to the Continued Fractions. Conference on Mathematics, Informatics, Information Technology, Application in Education, Pamporovo, 10-12 October 2018, 199-207. https://www.researchgate.net/publication/333245

[19] Doliwa, A. (2020) Non-Commutative Double-Sided Continued Fractions. Journal of Physics A: Mathematical and Theoretical, 53, Article ID: 364001.

[20] Ahmed, N., Alamri, S.Z. and Raseem, M. (2018) Probing Schrodinger Equation with a Continued Fraction Potential. NRIAG Journal of Astronomy and Geophysics, 7, 1-3. https://doi.org/10.1016/j.nrjag.2018.03.001

[21] Dani, S.G. and Nogueira, A. (2014) Continued Fractions for Complex Numbers and Values of Binary Quadratic Forms. Transactions of the American Mathematical Society, 366, 3553-3583. https://doi.org/10.1090/S0002-9947-2014-06003-0

[22] Nieuwveld, J. (2021) Fractions, Functions and Folding; a Novel Link between Continued Fractions, Mahler Function and Paper Folding. M.Sc. Thesis, Radboud University, Nijmegen. 